\title{
Contact-line dynamics and damping for oscillating free surface flows
}

\author{
Lei Jianga) \\ RA3-254, Logic Technology Development, Intel Corporation, 2501 NW 229th Avenue, Hillsboro, \\ Oregon 97124 \\ Marc Perlin \\ Department of Naval Architecture and Marine Engineering and Department of Mechanical Engineering, \\ University of Michigan, Ann Arbor, Michigan 48105 \\ William W. Schultz \\ Department of Mechanical Engineering and Department of Naval Architecture and Marine Engineering, \\ University of Michigan, Ann Arbor, Michigan 48105
}

(Received 12 September 2003; accepted 5 December 2003; published online 4 February 2004)

\begin{abstract}
New experimental data on the frequency and damping of Faraday water waves in glass tanks are presented to demonstrate the contact-line effect on free surface flows. We find a complicated nonlinear relationship between wave frequency and amplitude near contact lines: The amplitude dispersion for decaying standing waves directly progresses from a nonlinear regime due to large amplitude to a regime due to contact-line nonlinearity. The relative damping rate is also a function of the wave amplitude, increasing significantly at smaller wave amplitude. These results are discussed in relation to different formulations of contact-line conditions for oscillatory motions and free surface flows. A new model is proposed to explain the observed amplitude scaling in the frequency and damping rate, and to relate these behaviors to slip-length and other contact-line measurements by Ting and Perlin [J. Fluid Mech. 295, 263 (1995)]. (C) 2004 American Institute of Physics. [DOI: 10.1063/1.1644151]
\end{abstract}

\section{INTRODUCTION}

It is well known that the static shape of a fluid free surface near a solid surface is determined by the gravity (or other potential force) and wetting force balance. In general, the contact angle can be described by Young's equation for a balance between the surface energies of the three phases. In a dynamic configuration, however, additional viscous, inertia, and surface forces (e.g., electrostatic, van der Waals) determine the contact-line motion and the free surface near the contact line. There have been an increasing number of studies of the role of wetting forces and various spreading and dewetting phenomena. Herein, we are interested in the effect of "macroscopic" contact angle and its interaction with free surface flows, not the complex dynamics of the three-phase contact line on the nanometer scales. We further restrict ourselves to some interesting nonlocal effects of contact line dynamics on oscillating flows.

Most previous studies on contact line dynamics have focused on low-Reynolds number, unidirectional flow where viscous and capillary forces dominate. For simplicity, it is often assumed that the flow is unidirectional, such as in droplet spreading. With these assumptions, Dussan ${ }^{1}$ and subsequent studies (e.g., $\mathrm{Cox}^{2}$ ) introduced slip conditions to remove the contact-line singularity and derived equations that relate contact angle with contact-line velocity. In many cases, a lubrication approximation is an accurate representation of local fluid dynamics $\left(\right.$ Greenspan $\left.^{3}\right)$ if the contact angle is

a)Electronic mail: lei.jiang@intel.com small. Near the contact line this approximation avoids the singularity in the Stokes-flow formulation and simplifies the contact angle dynamics to the form $\theta \sim \mathrm{Ca}^{1 / 3}$. This is often referred to as Tanner's law, ${ }^{4}$ where Capillary number Ca represents the viscous-to-capillary force ratio and is proportional to contact-line relative velocity. For brevity, we refer to more comprehensive reviews ${ }^{5-8}$ of these models for contact-line dynamics.

Although we focus on oscillating (unsteady) flows with contact-line effects where these models do not directly apply, the scaling of capillary and viscous effects can be similar with those of unidirectional flows. Thus we want to explore common features and differences between wetting behaviors in oscillatory versus unidirectional flows. Through our experiments and comparison with previous analysis, we find such scaling similarity exhibited in both local (contact-line) and nonlocal (frequency and damping) dynamics.

This paper is organized as follows. We first review the contact-line models and analysis for oscillatory flows and surface waves in Sec. II. In Sec. III, we describe some interesting observations and experimental analysis of surface water waves in a container, mainly the wave frequency and damping in various wave tanks and wetting conditions. These data are compared with both a scaling model and more detailed analytical and numerical analysis in Sec. IV.

\section{REVIEW}

\section{A. Models for oscillatory contact lines}

Contact-line oscillation has been studied by Young and Davis, ${ }^{9}$ with the assumption that the contact-line motion is 

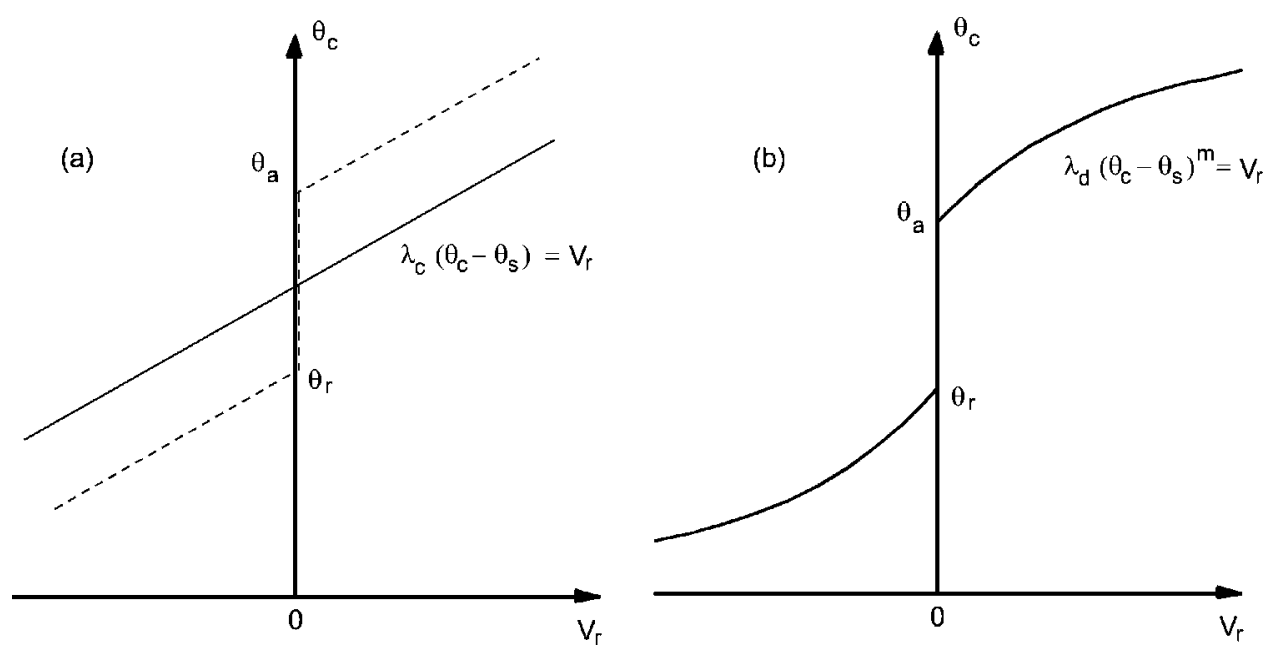

FIG. 1. Illustrative contact-line models with contact angle vs contact-line velocity. (a) The linear model of Hocking (Refs. 10 and 11) with (dashed line) and without hysteresis. (b) The model used for droplet spreading analysis (Dussan-Ref. 5, de Gennes-Ref. 7) with $m=3$ as the usual empirical exponent (consistent with lubrication analysis). local to the vicinity of the contact line and decoupled from bulk fluid motion. A Stokes flow model describes the viscous force that balances the capillary force, with contact-angle hysteresis included. Hocking ${ }^{10,11}$ then postulated that the dynamic contact angle $\theta_{d}$ is linearly proportional to the contact-line velocity $V_{r}$, as shown in Fig. 1(a). Adopting a "macroscopic" contact angle assumption, he approximated the local free surface slope as $\eta_{x}=\theta_{d}-\pi / 2$ and used its relation to free-surface velocity to specify a "contact-line" condition for free surface flow:

$$
\eta_{t}=c \eta_{x},
$$

where $\eta$ is the surface elevation at the contact line in fixed coordinates, the $x$ axis is perpendicular to the solid surface. The coefficient $c$ has the units of velocity. The contact angle $\theta_{d}$ and the contact-line relative velocity $V_{r}$ are represented by $\eta_{x}+\pi / 2$ and $\eta_{t}$ (with fixed wall), respectively. To simplify this formulation, we assume a $\pi / 2$ static contact angle.

Using (1), Hocking ${ }^{10,11}$ calculated the contact-line damping and meniscus waves caused by an oscillating plate in a liquid. In studies by Miles, ${ }^{12-14}$ the coefficient $c$ is made dimensionless with capillary length $l=(\sigma / \rho g)^{1 / 2}$, i.e., $\lambda_{c}$ $=c / \omega l$, where $\omega$ is the oscillation frequency. Comparison of (1) with contact-line models of unidirectional flow shows that the coefficient $\lambda_{c}$ is a parameter similar to slip length $l_{s}$. In the Navier slip condition, $l_{s}$ is defined as the slip velocity divided by the shear at the wall. ${ }^{15}$ Miles $^{13}$ showed that when the Navier slip condition models the contact-line region, the coefficient $\lambda_{c}$ is directly proportional to slip length $l_{s}$. Hereafter, we denote $\lambda_{c}$ as the "slip coefficient" specific to contact-line conditions of type (1). Miles ${ }^{13}$ further suggested that the slip coefficient can be complex for an oscillatory contact line, representing certain phase offset between contact-line motions and flow motions. He derived both damping and frequency shifts for linear waves that are functions of the coefficient $\lambda_{c}$, but not a function of the wave amplitude (i.e., linearity was assumed).

\section{B. Experiments on damping and frequency shift}

Keulegan ${ }^{16}$ studied the damping of surface standing waves with several liquids, in containers of different sizes, and with different container material. The total damping was measured by recording wave amplitude with time, and was separated into one component related to the viscous effect and another related to the surface tension, based on dimensional analysis. Although there is a good correlation between the damping coefficient and surface tension, Keulegan found it difficult to explain the drastic increase in damping when switching from a glass container to one constructed of Lucite $^{\mathrm{TM}}$. However, he pointed out that water wets Lucite much less than glass. Keulegan also observed an interesting dependence of damping on wave amplitude by comparing damping estimated from a different number of wave cycles $n$ after wave excitation is stopped. Damping for large $n$ (thus smaller amplitude) is considerably larger than that for small $n$ (larger amplitude). It was hypothesized that this "anomaly mode" is related to how water (or other liquids) wets the surface and related to "meniscus deformation." We will discuss this in more detail with our experiments. In summary, Keulegan ${ }^{16}$ was the first to notice that any effect of wetting (or contact-line motion) on damping is also amplitude dependent.

Cocciaro et al. ${ }^{17}$ also examined frequency and damping of standing waves and found discrepancies with linear wave theory that can be explained partially as contact-line effects. For waves in a Plexiglas ${ }^{\mathrm{TM}}$ circular cylinder with a static contact angle of $\theta_{s}=62^{\circ}$, the frequency and damping rates are also functions of the wave amplitude, as in the observation of Keulegan. Cocciaro et al. ${ }^{17}$ proposed that Hocking and Miles' theories would match experimental data if the coefficient was proportional to the wave amplitude.

\section{Experiments on contact-line oscillations}

Ting and Perlin ${ }^{15}$ studied contact-line dynamics with high-speed imaging near a vertically oscillating plate immersed in water. Detailed measurements again revealed a complex contact angle versus contact-line speed relation, with strong hysteresis and clear vortical motions in the vicinity of the contact line [Fig. 2(a)]. Using a formulation similar to Hocking and Miles, they extracted a slip length that has a nonlinear dependence on oscillation amplitude. 
(a)
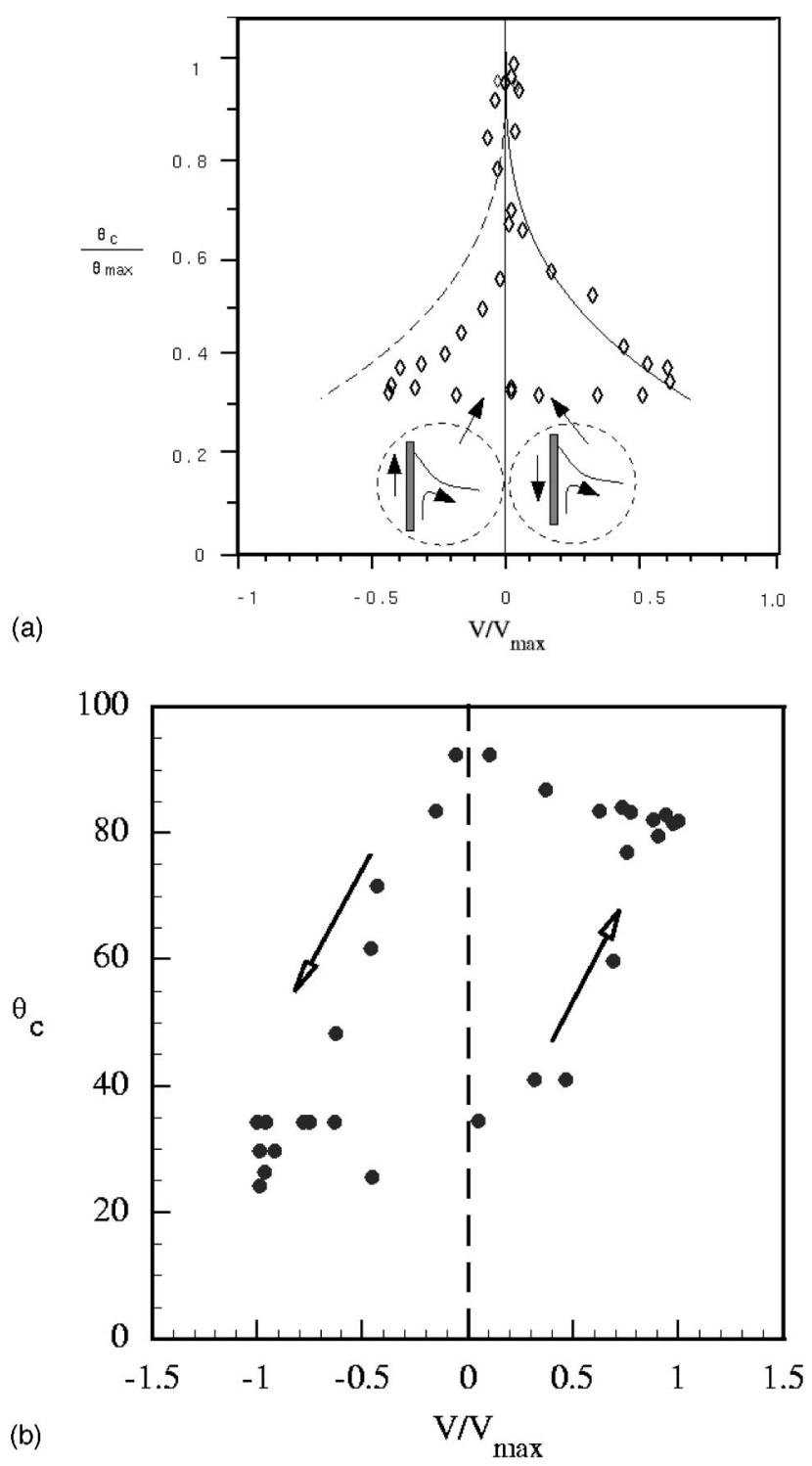

FIG. 2. (a) Relative contact angle vs contact-line velocity for a $2 \mathrm{~Hz}$ oscillation (Ting and Perlin-Ref. 15). The insets demonstrate the corner vortex formed at the maximum plate positions ( $\mathrm{Stroke}=3 \mathrm{~mm}$ ). (b) Extracted contact angle vs contact-line velocity data based on Cocciaro et al. (Ref. 17).

Note that the observations from Cocciaro et al. ${ }^{17}$ also suggest a dependence of slip length on wave amplitude, albeit a linear one. However, the hysteresis behavior is very different, as shown in Fig. 2(b).

As mentioned earlier, models for low-Reynolds number, unidirectional contact-line motion (e.g., drop spreading) usually result in a cubic relation between contact angle and velocity. This can be explained based on a lubrication approximation with and without slip, for the condition $\mathrm{Ca} \ll 1$ (Cox, ${ }^{2}$ Hocking ${ }^{18}$ ), and verified with experimental data (see Ehrhard and Davis, ${ }^{19}$ and references therein). Here, the capillary number $\mathrm{Ca}=\mu U / \sigma$ represents the ratio of the viscous force to the surface tension force. By analogy, a nonlinear relation between contact angle and velocity for oscillatory flow is reasonable and consistent with the experiments of Ting and Perlin ${ }^{15}$ under the same assumption of $\mathrm{Ca} \ll 1$ (because of the small Weber number), perhaps even for high Reynolds number cases.

\section{EXPERIMENTS}

\section{A. Setup and analysis procedures}

The following results on wave damping and frequency shift were obtained with the Faraday wave setup that we used to study nonlinear standing water waves (Jiang et al. ${ }^{20,21}$ ). In those studies, a complex demodulation technique was used to extract the accurate frequency of the time series measured by a wave probe. It was already known ${ }^{20}$ that the excitation frequency of Faraday waves and its hysteresis are strong functions of the contact-line behavior of water with the container wall. For example, addition of Photo-Flo, a common wetting agent, reduces the hysteresis significantly, and without such an agent, the natural frequency as determined from a neutral stability map for Faraday waves is higher than that predicted from linear stability theory with negligible contactline effects. These results effectively correspond to smallamplitude waves, as the excited waves are near the neutral stability boundary.

Using the same experimental setup and probe measurement technique ${ }^{20,21}$ we also excite high-amplitude standing waves by Faraday resonance, and then remove or gradually reduce the vertical forcing signal and measure the wave decay over time. The complex demodulation, when applied to a decaying wave signal, can also be used to extract the slowly varying amplitude and the frequency. Henderson et al. ${ }^{22}$ derived the following expression for the decaying wave elevation:

$$
\eta=e^{-\gamma t}\left[A(x) \cos \left(\omega_{f}-\Delta \omega\right) t+B(x) \sin \left(\omega_{f}-\Delta \omega\right) t\right],
$$

where $\omega_{f}$ is the forced wave frequency (half the forcing frequency in Faraday resonance) and $\Delta \omega$ is given by

$$
\Delta \omega=\frac{\omega_{f}^{2}-\omega_{n}^{2}}{\omega_{f}},
$$

where $\omega_{n}$ is the natural frequency. Since the forcing frequency is known and can be "demodulated" from the original signal, we then estimate the actual wave frequency based on the above formula. The digital filter is described elsewhere. ${ }^{21}$

\section{B. Frequency and damping of standing waves in rectangular tanks}

Figure 3 shows the primary mode of an excited wave in the narrow tank setup as used in our previous studies. The wave probe is situated at the center of the tank to capture the largest wave elevation. The small aspect ratio (1:10) is chosen to ensure the longitudinal mode excitation and minimal interference of the sidewall on wave probe accuracy. A typical decaying signal is shown in Fig. 4(a) with the forcing signal terminated at time $t=5 \mathrm{~s}$. The length of the remaining time series is $\sim 35 \mathrm{~s}$, and the sampling frequency is $300 \mathrm{~Hz}$ (much larger than the wave frequency of $\sim 1.6 \mathrm{~Hz}$ ). The original time series contains higher harmonics and noise for 


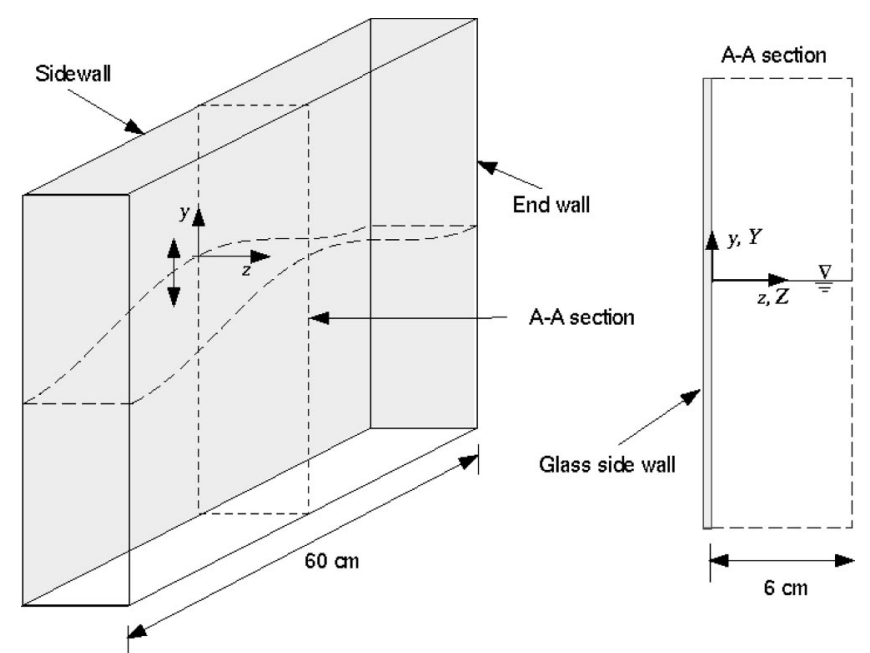

FIG. 3. Our coordinate system for standing waves in a narrow rectangular tank. The contact-line close up and coordinate $s$ used in the following analysis is shown in section A-A. The fundamental wave model is shown with the dashed line representing the contact lines (two side walls are the main contact-line effect contribution because of the perimeter ratio). two reasons: (1) a nonanalog filter was applied, (2) the elevation signal was truncated at $0.001 \mathrm{~mm}$ (wave probe limit). Complex demodulation is applied to filter the higher harmonics and noise first, and to obtain the first-harmonic amplitude and phase as shown in Figs. 4(b) and 4(d). The demodulation frequency is set at $1.6 \mathrm{~Hz}$ (forcing frequency). The damping rate is estimated from the instantaneous slope of the log-wave amplitude ratio to give us a more complete history of damping as a function of time and therefore wave amplitude. Figure 4(c) shows interestingly that the damping ratio (slope) is more nonlinear as wave amplitude becomes smaller, an observation to be discussed in more detail later.

Figure 5 shows the extracted natural wave frequency versus instantaneous wave amplitude during the decay that can be clearly divided into three regimes. Immediately after forcing is stopped, both phase and amplitude show a transition of 2-3 s. During this transition, the wave frequency switches to that of free waves, thus lower than the forcing frequency because of finite wave amplitude. This is consistent with the second regime, where the nonlinear wave fre-
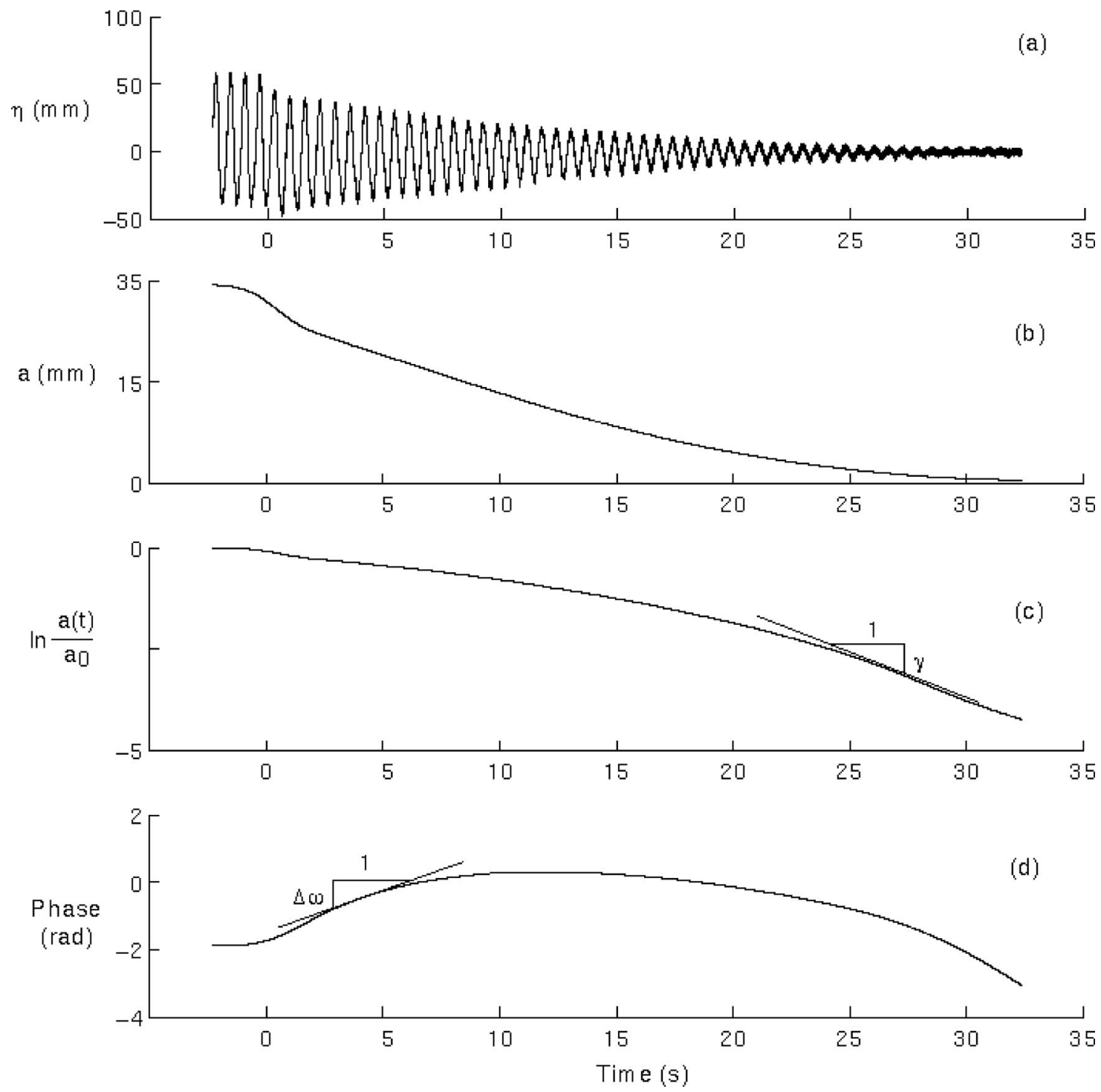

FIG. 4. (a) Decaying surface elevation signal, (b) the complex-demodulated amplitude of the first harmonic fundamental wave mode), (c) the wave amplitude decay (damping rate), and (d) the phase angle of the first harmonic. The initial forcing amplitude $F$ is $4.3 \mathrm{~mm}$, the forcing frequency is $3.2 \mathrm{~Hz}$ (first-harmonic amplitude of $35 \mathrm{~mm}$ ). 


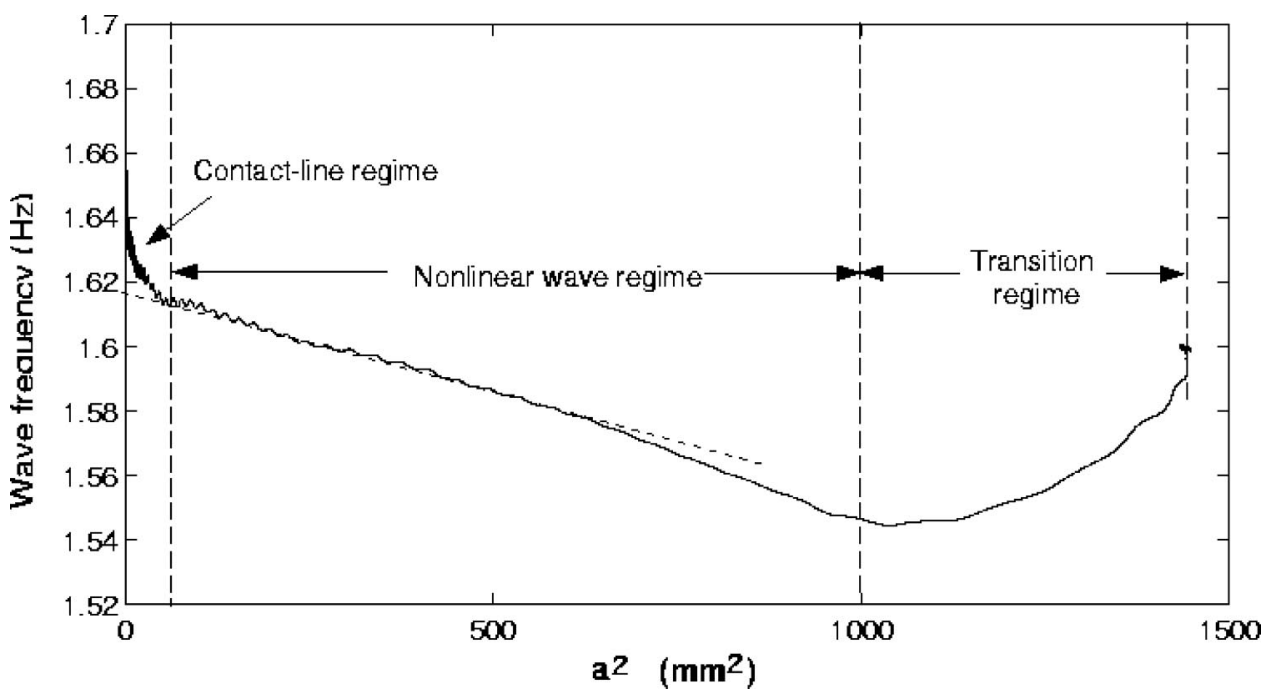

FIG. 5. Cyclic wave frequency $\omega / 2 \pi$ vs the square of the wave amplitude square (first harmonic) during the wave decay. quency is approximated by the dispersion relation (for moderate wave amplitude):

$$
\omega=\omega_{n}\left[1-\omega_{2}(k a)^{2}\right],
$$

where $k$ is the primary wavenumber and $a$ is the wave amplitude. The natural frequency is denoted as $\omega_{n}$ in $\mathrm{rad} / \mathrm{s}$ or $f_{n}=\omega_{n} / 2 \pi$ in Hz, based on linear wave theory, and $\omega_{2}$ is the second-order correction to wave frequency, a constant based on Stokes wave theory. Therefore the frequency increases linearly with decrease in dimensionless wave amplitude $(k a)^{2}$, as shown in Fig. 5. Extrapolation of the frequencyamplitude dependence in this regime to zero amplitude corresponds to $f_{n}=1.615 \mathrm{~Hz}$, very close to the theoretical estimate $(1.611 \mathrm{~Hz})$. We can infer that the contact-line effect is not important in this regime because of the moderate wave amplitude (sidewall most of the time is coated by a receding water film with large fluid displacement), and hence a freeedge condition (implicit assumption of standard linear wave theory) is valid. The small, high-frequency oscillation is attributed to digitization error and interaction with the digital filter. An averaging procedure removes such oscillation in subsequent data analysis. Decaying time series with excitation amplitude and frequency shows $\omega_{2}$ ranging from 0.34 to 0.47 , but the extrapolated frequency $f_{n}$ at zero amplitude is consistently $\sim 1.615 \mathrm{~Hz}$ from (4) for this amplitude regime.

The more interesting regime is the one close to the small amplitude limit: There is an unexpected rapid decay increase with further reduction of wave amplitude. To examine this regime in more detail, the same data set is plotted in Fig. 6 on a $\log -\log$ graph. Three different time series with different forcing amplitude in fact demonstrate the same asymptotic behavior, although those for $F=4.0 \mathrm{~mm}$ (displacement amplitude associated with the vertical forcing) show a larger oscillation of unknown cause. Separately, neutral stability experiments $^{20}$ also revealed an actual wave frequency $f_{n}$ $=1.662 \mathrm{~Hz}$; higher than the linear theory prediction. Therein it was attributed to strong contact-line on the sidewalls as a result of the narrow 1:10 tank geometry. However, this decaying time series indicates there is no fixed wave frequency even in this small wave amplitude limit. Figure 6(a) clearly shows a $-2 / 3$ power law between frequency shift and wave amplitude (for $0.5 \mathrm{~mm}<a<7 \mathrm{~mm}$ ), as in Fig. 6(b) for the average of the three time series. This trend is clear except for the smallest wave amplitude recorded (limited by the length of the time series, not by the wave probe capability).

Although the frequency-amplitude correlation is classified as three regimes in the above analysis, the transitions between regimes are smooth. We note that at no time during the decay is the frequency constant as predicted by linear theories. The amplitude dispersion directly progresses from a nonlinear regime due to large-amplitude nonlinearity to another nonlinear regime with even stronger dependence on amplitude to the smallest resolvable amplitude. One possible explanation for the overlapping nonlinear regimes is the different parameters governing the nonlinearity: The first regime is dependent on wave steepness $k a$ while the wave amplitude ratio to capillary length may be the determining factor of nonlinearity in the second regime at low amplitude (e.g., see Faraday waves in cylinder tanks in Sec. III C).

Similarly, we can extract the damping rate (viscous and contact-line) dependence on wave amplitude, as shown in Fig. 7. The high-frequency oscillation appears larger, particularly for the series obtained with smaller amplitude forcing $(4.0 \mathrm{~mm})$. All three, however, follow approximately a $-1 / 3$ power law during the transition from the nonlinear to the contact-line regime, and increase from 0.05 to a maximum of $0.25 \mathrm{~s}^{-1}$, a fivefold increase (wave amplitude $1.3 \mathrm{~mm}<a$ $<7 \mathrm{~mm}$ ). This is consistent with higher damping due to contact-line effects. The damping rate decreases for even smaller amplitudes $(a<1.3 \mathrm{~mm})$, suggesting a change in the contact-line behavior (possibly from a moving contact line to a pinned one, as discussed by Cocciaro et al. ${ }^{17}$ ). Data fitting indicates a $1 / 3$ power-law exponent, although it is for only a short span at the end of the time series.

\section{Surfactant, viscous, and tank geometry effects}

Although we demonstrated consistency with previous experiments regarding the contact-line effect on the fre- 

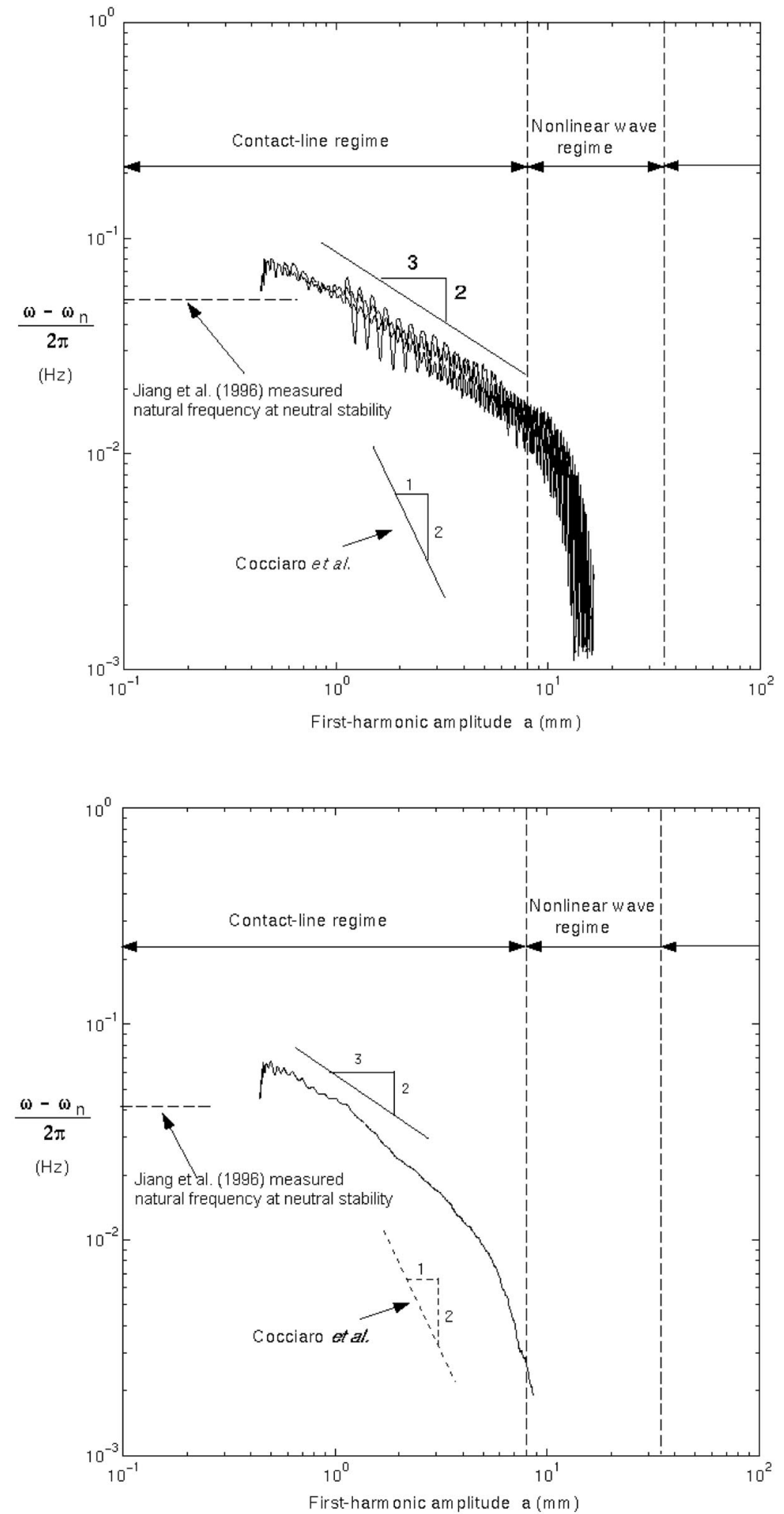

FIG. 6. Cyclic frequency difference $\left(\omega-\omega_{0}\right) / 2 \pi$ vs wave amplitude $a$ for the contact-line regime. Initial displacement amplitude associated with the forcing, $F$ $=4.0,4.3$, and $4.6 \mathrm{~mm}$. (Bottom): Combined dependence of frequency on amplitude (average of three series with filtering of high-frequency signal). quency shift and damping, we also observe that the nonlinearity in frequency and damping was not due solely to viscous or static contact angle effects. Henderson et al. ${ }^{22}$ conducted standing wave experiments where the static contact angle was varied (from hydrophobic to hydrophilic) by pairing different wall solid material and working liquid. Fre- quency was found to increase with contact angle, and damping was found to decrease with increasing contact angle. It is possible that the higher damping at small wetting angle (similar to water-on-glass as in our experiments) could be similar to the observation described herein. However, the same theory does not seem to provide any explanation for 

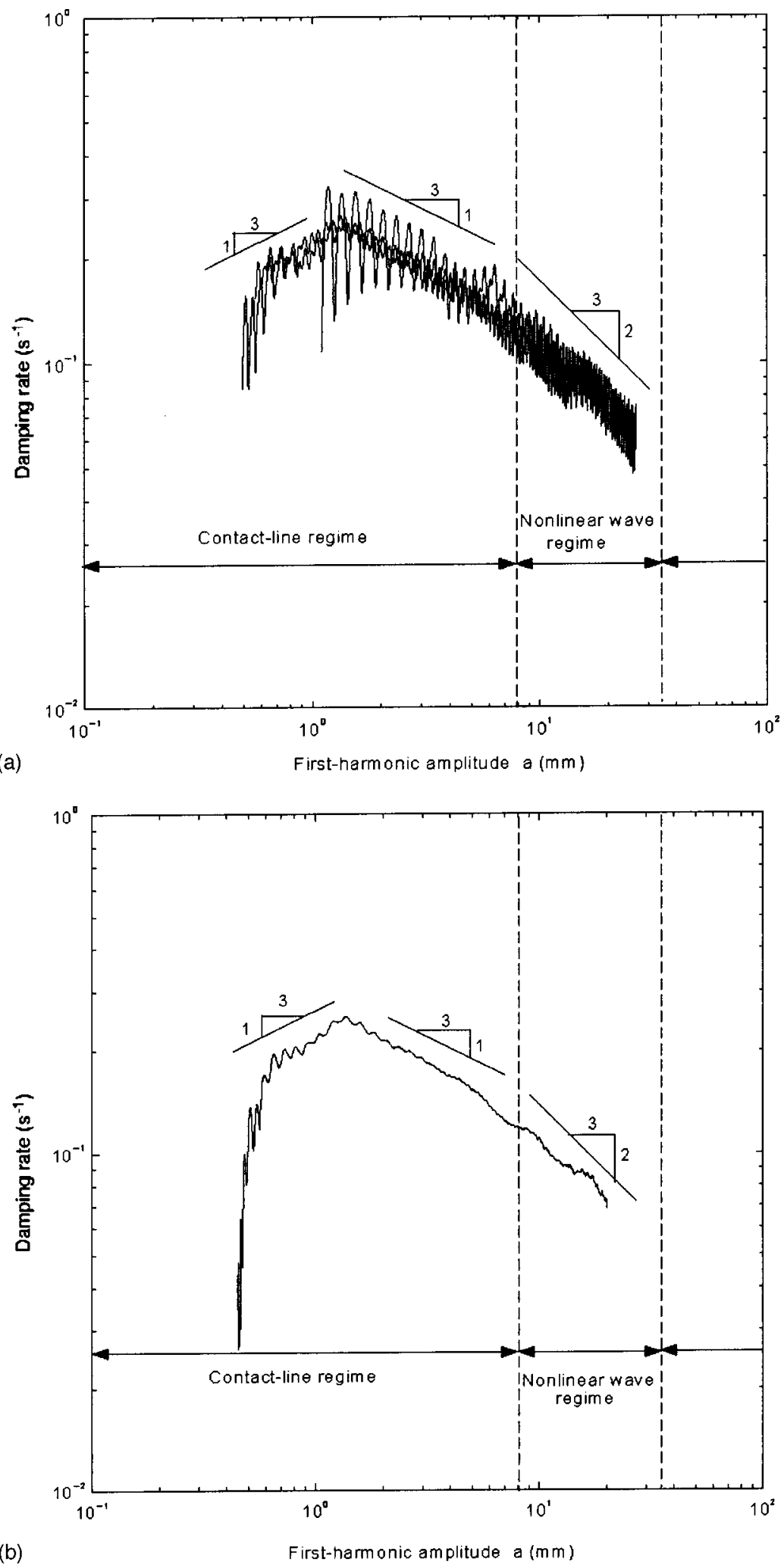

FIG. 7. Damping rate with (a) different initial displacement amplitude associated with the forcing ( $F$ $=4.0,4.3,4.6 \mathrm{~mm}$ ), and (b) averaged among the three series. the frequency dependence on amplitude observed herein. The smaller frequency change at hydrophilic conditions probably can be attributed to dissipation in the wetting film as explained by Miles in his viscous boundary-layer analysis for the zero-contact angle case (no hysteresis) that does not apply in our experiments.

Martel and Knobloch ${ }^{23}$ point out that an additional vis- cous mode need be considered as part of the bulk motion when analyzing damping. This viscous effect requires at least weakly nonlinear analysis, which could naturally introduce amplitude dependence, although different from the observed scaling herein. This additional effect can be the cause of the discrepancy in frequency between theory and experiments in Henderson and Miles. ${ }^{24}$ To verify if these effects (viscous 


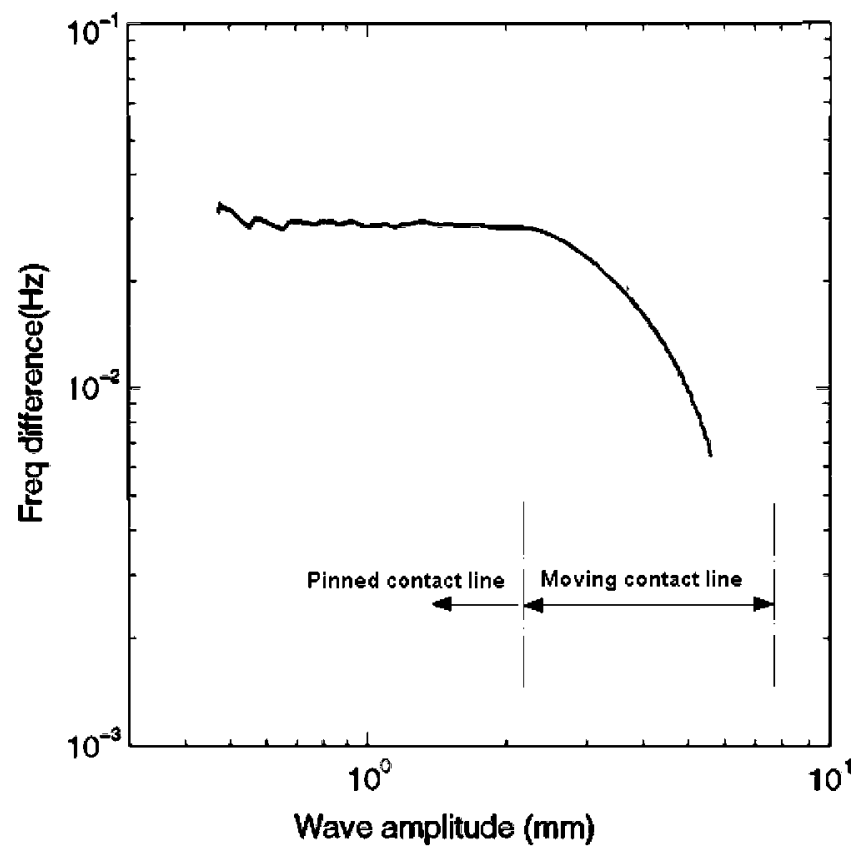

FIG. 8. Frequency shift for the axisymmetric mode in a circular tank with treated water.

and contact angle) contribute to the "anomaly" observed here, we conducted experiments using water mixed with Photo-Flo (lubricating film and wetting agent) with increased viscosity and reduced contact angle (see Jiang et al. ${ }^{20}$ for the viscosity data). The measurements suggest that the frequency of the surface elevation approaches a value close to the linear natural frequency (without contact-line effects) as amplitude approaches zero. However, in contrast to the treated water case, there is no clear amplitude dependence in either the frequency shift or the damping.

Additional experiments were conducted with Faraday waves (axisymmetric mode) in a small circular cylinder (Pyrex glass, $12.8 \mathrm{~mm}$ ) and a larger cylindrical cylindrical tank (Plexiglas $15.9 \mathrm{~cm}$ diameter). An example for the 15.9 $\mathrm{mm}$ tank with treated water is shown in Fig. 8. The frequency of the decaying elevation shows amplitude dependence at $a>2 \mathrm{~mm}$, while the frequency appears fixed for smaller amplitudes $(a<2 \mathrm{~mm})$. Note that for the rectangular tank, waves show amplitude-dependent frequencies as small as $0.5 \mathrm{~mm}$. This difference might be due to the smaller contact-line displacement in the cylinder for the same wave amplitude as compared to waves in the rectangular tank. For the axisymmetric mode in the circular tank, the maximum displacement is only $1 / 3$ to $1 / 2$ of the maximum wave amplitude measured at the tank center. At small amplitude $(<2 \mathrm{~mm})$, the contact line is effectively pinned at the tank wall. We did not conduct detailed verification of the wave profile near the wall for the cylindrical tank, and the record used to generate Fig. 8 may be too short to provide valuable information (i.e., amplitude dependence scaling) needed in the small-amplitude (contact-line dominated) range.

The natural frequency without contact-line and viscous effect is $2.537 \mathrm{~Hz}$ for the axisymmetric mode in the above example. The measured final frequency is $2.570 \mathrm{~Hz}$ with treated-water and $2.530 \mathrm{~Hz}$ with a Photo-Flo mixture. Again, the Photo-Flo addition provides a wetting film on the surface of the container that reduces the natural frequency closer to the linear wave frequency (without contact-line effects). This is qualitatively similar to Cocciaro et al. ${ }^{17}$ and to Henderson et $a .^{22}$ with regard to contact line observations. Addition of Photo-Flo produces the same effect as stated above for rectangular tanks - no clear amplitude dependence is observed in the frequency change or damping rate.

Any additional viscous mode as described by Martel and Knobloch $^{23}$ cannot be responsible for the differences between Faraday waves in treated-water versus those with a Photo-Flo mixture. In fact, higher viscosity with the PhotoFlo mixture corresponds to behavior more closely predicted by previous analysis based on linear wave theory. Rather, removal of the contact angle hysteresis, partial wetting, or other possible contact-line effects significantly reduces the "anomalous" frequency and damping behaviors (as a function of amplitude). We have no evidence these experiments apply to a broad range of wetting angles, although qualitative agreement with observations by Keulegan ${ }^{16}$ suggest common features of the contact-line dynamics on the partially wetted surface (contact angle significantly smaller than $90^{\circ}$ but nonzero).

As the above experiments with PhotoFlo and with circular cylinders show, the presence of corners in our narrow $10: 1$ rectangular tank is not likely to be the main cause for the amplitude dependence in frequency and damping. We note that earlier studies by Keulegan ${ }^{16}$ used several tanks with the same width-to-length ratio of 0.217 compared to 0.1 here. The damping increases toward smaller amplitude waves were observed in all cases, although the corner effect should be less dominant for larger tanks with smaller perimeter-to-area ratio. Although corner conditions may play a key role in pattern selection for Faraday resonance, ${ }^{26}$ the wave studied here is the fundamental mode with the tank length equal to wavelength (or in the case of Keulegan, half the wavelength). Therefore we expect limited corner effects on overall wave frequency and damping. However, the detailed contact-line dynamics or viscous contribution near corners can only be determined by additional experiments or theoretical analysis. Hereafter, we assume the corner effect is negligible and focus on the contact-line condition at the periphery of the rectangular tank, with its main contributions at boundaries in the $X-Y$ plane (Fig. 3).

\section{RESULTS AND DISCUSSIONS}

The fundamental question regarding formulation of the contact-line condition is to identify appropriate boundary conditions at the three-phase interface that are also physically reasonable, such as the introduction of slip to remove the singularity. There also exists a key difference between macroscopic contact angle and the microscopic one. For many cases, using the dynamic macroscopic contact angle (e.g., Dussan ${ }^{5}$ ) or even the static contact angle (e.g., Hocking $^{18}$ ) is sufficient to describe the outer fluid motion either numerically (by solving the Navier-Stokes equations) or analytically (asymptotic analysis). Very near the contact 
TABLE I. Various contact-line models and implications on frequency and damping scaling with wave amplitude.

\begin{tabular}{|c|c|c|c|c|c|}
\hline \multirow[b]{2}{*}{$\begin{array}{l}\text { Wave amplitude } a \\
\text { Contact-line freq } \\
\text { correction }\end{array}$} & \multirow{2}{*}{$\begin{array}{c}\text { Miles }^{\mathrm{a}} \\
\ldots \\
\sim \text { constant }\end{array}$} & \multicolumn{2}{|c|}{ Cocciaro et al. ${ }^{\mathrm{b}}$} & \multicolumn{2}{|c|}{ Present work } \\
\hline & & $\begin{array}{c}a<\omega l \\
\sim \text { constant }\end{array}$ & $\begin{array}{l}a>\omega l \\
\sim a^{-2}\end{array}$ & $\begin{array}{c}a<\omega l \\
\text { N/A }\end{array}$ & $\begin{array}{l}a>\omega l \\
\sim a^{-2 / 3}\end{array}$ \\
\hline $\begin{array}{l}\text { Contact-line } \\
\text { damping rate }\end{array}$ & $\sim$ constant & $\sim a$ & $\sim a^{-1}$ & $\sim a^{1 / 3}$ & $\sim a^{-1 / 3}$ \\
\hline $\begin{array}{l}\text { Contact line model } \\
\text { (dimensionless) }\end{array}$ & $\begin{array}{c}\eta_{t}=\lambda_{c} \eta_{x} \\
\lambda_{c}=\text { constant }\end{array}$ & \multirow{2}{*}{\multicolumn{2}{|c|}{$\begin{array}{l}\qquad \eta_{t}=\lambda_{c}(a) \eta_{x} \\
\qquad \lambda_{c} \sim a \\
\text { Ad hoc extension of } \\
\text { linear model (Miles) }\end{array}$}} & \multirow{2}{*}{\multicolumn{2}{|c|}{$\begin{array}{l}\text { (1) } \eta_{t}=\lambda_{c}(a) \eta_{x}, \lambda_{c} \sim a^{1 / 3} \\
\text { (2) } \eta_{t}=\lambda_{d}\left(\eta_{x}\right)^{3}, \lambda_{d} \sim \text { constant } \\
\text { (1) Ad hoc extension of Miles, }{ }^{a}{ }^{a} \text { (2) New contact line condition }\end{array}$}} \\
\hline Approach & $\begin{array}{l}\text { Boundary-layer } \\
+ \text { eigenvalue }\end{array}$ & & & & \\
\hline
\end{tabular}

${ }^{\text {aReferences } 12 \text { and } 13 .}$

${ }^{\mathrm{b}}$ Reference 17 .

line, either a Stokes flow analysis or a lubrication approximation can be used to describe the local angle-velocity relation that necessarily includes any slip model. Slip length can therefore be regarded as a key parameter linking the microscopic and macroscopic levels in contact-line dynamics.

Although the same approach should apply equally well to oscillating flows and free surface-wave analysis, an accurate and simple formulation of a contact angle-contact line model has been elusive, as all experimental and numerical analyses indicate much more complex behavior including hysteresis, nonlinearity, and unsteady dependence of angle versus velocity as observed by Cocciaro et al. ${ }^{17}$ and in more detail by Ting and Perlin. ${ }^{15}$ The hysteresis is explained partially by inertia and viscous effects and their interaction with flow in meniscus regions (e.g., corner vortex). Qualitatively similar hysteresis [Fig. 2(a)] was reproduced in numerical simulation by Dreyer (private communication) with FIDAP, a finite-element method solver for the Navier-Stokes equations. Therein a linear contact-line condition relating contact angle to contact-line displacement was used in addition to the Navier slip condition on the solid surface. However, it remains unresolved whether other aspects of the oscillating contact-line dynamics can be explained by inertial and viscous effects alone, i.e., the unsteady stick-slip motion and nonlinear dependence on amplitude.

An additional difficulty with applying general contactline conditions to free surface-wave analysis is the compatibility of these conditions with the eigenvalue problem formulation. For example, variational formulations are required to solve the weak form of the linear water wave problem with fixed contact line (Benjamin and $\mathrm{Scott}^{25}$ ), because direct eigenfunction expansion is not feasible with such boundary conditions. Other aspects of capillary effects for nonlinear waves are reviewed in more detail by Perlin and Schultz. ${ }^{26}$ For this work we will proceed based on previous linear analysis by Hocking and Miles, and focus on the timeaveraged contact-line behavior rather than the exact model that would explain time-dependent contact-angle variation in Ting and Perlin. ${ }^{15}$

Miles $^{12,13}$ showed that a boundary-layer approximation can be applied when the capillary length $l=(\sigma / \rho g)^{1 / 2}$ $(=2.7 \mathrm{~mm}$ for a pure, air-water interface) is much smaller than the wavelength. Applying the contact-line condition (1) resulted in a first-order frequency model

$$
\frac{\omega^{2}-\omega_{n}^{2}}{\omega_{n}^{2}}=\frac{\Gamma}{1-i \lambda_{c}},
$$

where $\Gamma$ is a constant form factor determined by the wave mode, and the "slip coefficient" $\lambda_{c}$ is dimensionless. For the fundamental mode with a wavelength of $60 \mathrm{~cm}$ in our experiments (10:1 tank aspect ratio), the form factor is $(4 / a$ $+2 / b) l=0.108$ with $a=60 \mathrm{~cm}, b=6 \mathrm{~cm}$. For $\lambda_{c}$ approaching zero and infinity, the above equation predicts frequencies for fixed and perfectly wetted contact lines, respectively. When the difference between the actual frequency and the natural frequency (without contact-line effect) $\omega_{n}$ is small, the above formulas can be reduced to

$$
\gamma_{c}=\frac{\Gamma \omega_{n} \lambda_{c}}{2\left(1+\lambda_{c}^{2}\right)}
$$

and

$$
\omega-\omega_{n}=\frac{\Gamma \omega_{n}}{2\left(1+\lambda_{c}^{2}\right)}+\frac{\gamma_{c}^{2}}{2 \omega_{n}},
$$

where $\gamma_{c}$ represents the imaginary frequency components, i.e., damping rate from the contact-line condition (1). The boundary-layer assumption is applicable to frequency estimates as long as the contact-line effect is confined within a capillary length $l$, as verified with the alternative approach using a variational formulation by Miles. The meniscus effect is also expected to be small, as estimated in Miles. ${ }^{12}$

Linearity of (1) is essential to ensure separable partialdifferential equations in Miles' boundary-layer and eigenvalue approach. Cocciaro et al. ${ }^{17}$ assumed that the amplitude dependence of wave frequency and damping can be represented by simply replacing the constant $\lambda_{c}$ with relation $\lambda_{c}$ $\sim a$. Applying this scaling in (6) and (7) gives the power law dependence observed in the experiments of Cocciaro et al. A similar argument suggests that our observed power-law dependence on amplitude warrants an assumed scaling of $\lambda_{c}$ $\sim a^{1 / 3}$. In other words, an ad hoc approach to fitting this linear model suggests that the scaling $\lambda_{c} \sim a^{1 / 3}$ would repro- 
duce the observed $-2 / 3$ and $-1 / 3$ scaling in frequency shift and damping, respectively. Comparison of these conditions is summarized in Table I.

The approach used by Cocciaro et al. and in our extension (model 1) is only ad hoc in that the nonlinear "slip coefficient" $\lambda_{c}(a)$ violates the assumptions in the linear eigenvalue approach of Miles, ${ }^{12,13}$ as does our conjecture (model 2) with nonlinear dependence of contact angle on contact-line velocity. A more general form of the proposed second model is

$$
\left|V_{r}\right|=\lambda_{d}\left|\left(\theta_{e}-\theta_{c}\right)\right|^{3},
$$

where $\theta_{e}$ is the static contact angle. We propose this second model as a more appropriate condition for frequency and damping analysis for the following three reasons. (Herein, $\theta_{e}$ merely represents the contact angle corresponding to zero velocity, while real static contact angle varies between the advancing and receding angles because of hysteresis.)

First, as explored in Hocking, ${ }^{10,11}$ a "slip coefficient" is proposed to incorporate fundamental slip behavior at the contact line, a physical behavior occurring at very local scale, not at the "macroscopic" contact angle level considered here. Therefore we expect a better contact-line model will adhere to the same principle of constant "slip coefficient" that represents microscopic dynamics in our macroscopic/continuum model, even though it is not straightforward to relate our second conjecture to Miles' analysis and demonstrate the $-2 / 3$ amplitude dependence in the frequency shift.

Second, the formulation of the second conjecture is similar to many studies of contact-line behavior in unidirectional wetting/dewetting flows as reviewed in the introduction, and is qualitatively consistent with the experimental data of Ting and Perlin. ${ }^{15}$ Contact-line models and experiments for lowReynolds number flows often lead to a cubic relation $U$ $\sim \Lambda \theta\left(\theta^{2}-\theta_{e}^{2}\right)$, where $U$ is the contact-line velocity and $\Lambda$ is only a function of fluid properties, surface tension, and viscosity. ${ }^{5,27,28}$ For small contact angle $\theta_{e}$, this is analogous to conjecture (2) in Table I $\left(U \sim \Lambda \theta^{3}\right)$. The mechanism behind Tanner's law $^{2}$ and (8) is probably the same, i.e., the contact-angle variation is caused by hydrodynamics very close to the contact line, and can be described by a balance between capillary and viscous forces locally. For example, when applying the boundary-layer analysis proposed by Miles, ${ }^{12}$ a stronger coupling with capillary forces might need to be included in the first-order viscous boundary-layer calculation. However, the mathematical analysis will be addressed in a subsequent paper.

When capillary effect is absent, Mei and $\mathrm{Liu}^{29}$ identified the meniscus corner as a key energy transfer region between bulk flow and wall boundary layers in their study of gravitywave damping. The importance of contact-line region is analyzed in even earlier study ${ }^{30}$ on damping-Miles used an energy dissipation estimate to calculate damping rate that emphasized an energy balance at the meniscus corner. We can apply a similar argument and assume that Young's force at the contact line, $F_{\mathrm{Y}}$, is proportional to $\sigma\left(\cos \theta_{c}-\cos \theta_{e}\right)$. This capillary force is balanced entirely by the viscous force locally, and therefore the dissipation over one wave cycle is then proportional to $F^{*} a$ where $a$ is again the wave amplitude. Using (8) for a small angle we obtain $F_{\mathrm{Y}} \sim \theta^{2} \sim V_{r}^{2 / 3}$. If we further assume that $V_{r} \sim \omega a$, the damping rate can be estimated by

$$
\gamma=\frac{F_{\mathrm{Y}} a}{\text { Energy }} \sim \frac{a^{2 / 3} a}{a^{2}} \sim a^{-1 / 3},
$$

i.e., the amplitude dependence measured in our Faraday wave damping rate. The contact line is a dominant dissipation source If these assumptions are correct. However, it is unclear how the same hypothesis will result in the frequency scaling we observed when capillary and contact-line effects are included.

Finally, we note that one of the most important results of Ting and Perlin ${ }^{15}$ is that the slip coefficient $\lambda$ has quadratic dependence on stroke amplitude in the plate-driven contactline oscillation (Fig. 25 therein). Since the slip coefficient therein is based on Hocking's model and defined as velocity divided by contact-angle variation, the time-averaged slip coefficient should be proportional to slip length, a microscopic parameter that should be independent of wave amplitude. Interestingly, when (8) is used, we note that the slip coefficient $\lambda_{c}$ in Fig. 25 of Ting and Perlin is equivalent to $\lambda_{d}\left(\theta_{e}-\theta_{c}\right)^{2}$. For a stroke amplitude less than $5 \mathrm{~mm}$, the contact-angle variation $\left(\theta_{e}-\theta_{c}\right)$ is approximately linearly proportional to amplitude in their measurement. ${ }^{15}$ Therefore the quadratic dependence of the slip coefficient $\lambda_{c}$ on amplitude is in fact consistent with Eq. (8) with a fixed $\lambda_{d}$. Equation (8) also matches qualitatively the contact-angle-velocity curve for one oscillation cycle, as shown in Fig. 2(a), but does not represent the important hysteresis effects. The actual model for the contact line is likely to be much more complex, although (8) might be a good representation of the time-averaged behavior (without the hysteresis). Contact-line characteristics (hysteresis, stick-slip, and slip) for highReynolds number oscillating flows were recently analyzed by Perlin, Schultz, and Liu (to be published) with comparisons of common features between different static contact angles.

\section{CONCLUSIONS}

We have shown that the frequency and damping of free surface waves wave can be determined accurately using a Faraday-wave tank experimental setup. In particular, using complex demodulation techniques permits finer resolution in frequency and damping extraction from a decaying wave signal. We find that the frequency of waves generated in a narrow glass tank with treated water increases with smaller wave amplitude rapidly during the initial decay, consistent with weakly nonlinear theory. With further decay in wave amplitude, however, the frequency continues to increase above the linear frequency, following a $-2 / 3$ power dependence on wave amplitude. The extrapolated frequency for infinitesimal waves is consistent with prior observation of contact-line induced frequency increase (Jiang et al. ${ }^{20}$ ), but the dependence on amplitude is new. Similarly, the damping rate also follows a power law dependence on wave amplitude in the same regime, with a $-1 / 3$ exponent. The maximum 
damping rate is reached at about $1 \mathrm{~mm}$ amplitude and the damping rate subsequently decreases with wave amplitude following a $1 / 3$ power law.

Our measurements of damping rate and frequency are consistent with observation by Keulegan. ${ }^{16}$ The techniques used here allow a more accurate determination of amplitude dependence that is different from results obtained by Cocciaro et al. ${ }^{17}$ We verify that the increased damping and frequency toward small amplitude are due to contact-line effect in a partial wetting condition, as addition of Photo-Flo removes such dependence. The increase in damping and frequency are consistent with the contact-line models of Hocking and Miles, although none predicted the observed amplitude dependence.

We have combined these new data with earlier work of Ting and Perlin ${ }^{15}$ to show that the frequency and damping dependence on amplitude is partially consistent with complex, nonlinear contact-line behavior for oscillatory flows. The scaling observed here indicates a contact-line condition that represents a local capillary-viscous force balance similar to model analysis of unidirectional and low-Reynolds number flows. We show that the proposed contact-line model explains at least the observed damping rate scaling with amplitude, and matches the time-averaged slip coefficient measurement in Ting and Perlin. ${ }^{15}$

\section{ACKNOWLEDGMENTS}

We are grateful for support of this work by the Office of Naval Research, and the National Aeronautics and Space Administration (Microgravity Program). We owe thanks to Chaolung Ting for his help in the experiment setup for this study and for his expertise in analysis of Faraday waves. We thank Z. Liu, M. Dreyer, and M. Miksis for discussions and suggestions.

${ }^{1}$ E. B. Dussan V., "The moving contact line: The slip boundary condition," J. Fluid Mech. 77, 665 (1976).

${ }^{2}$ R. G. Cox, "The dynamics of the spreading of liquids on a solid surface. 1. Viscous flow," J. Fluid Mech. 168, 169 (1986).

${ }^{3}$ H. P. Greenspan, "On the motion of a small viscous droplet that wets a surface," J. Fluid Mech. 84, 125 (1978).

${ }^{4}$ L. H. Tanner, "The spreading of silicone oil drops on horizontal surfaces," J. Phys. D 12, 1473 (1979).

${ }^{5}$ E. B. Dussan V., "On the spreading of liquids on solid surfaces: Static and dynamic contact lines," Annu. Rev. Fluid Mech. 11, 371 (1979).
${ }^{6}$ S. H. Davis, "Contact-line problems in fluid mechanics," J. Appl. Mech. Trans. ASME 50, 977 (1983).

${ }^{7}$ P. G. de Gennes, “Wetting: Statics and dynamics," Rev. Mod. Phys. 57, 827 (1985).

${ }^{8}$ T. D. Blake, "Dynamic contact angles and wetting kinetics," in Wettability, edited by J. C. Berg (Dekker, New York, 1993), p. 251.

${ }^{9}$ G. W. Young and S. H. Davis, "A plate oscillating across a liquid interface: Effects of contact-angle hysteresis," J. Fluid Mech. 174, 327 (1987).

${ }^{10}$ L. M. Hocking, "The damping of capillary-gravity waves at a rigid boundary," J. Fluid Mech. 179, 253 (1987).

${ }^{11}$ L. M. Hocking, "Waves produced by a vertically oscillating plate," J. Fluid Mech. 179, 267 (1987).

${ }^{12}$ J. W. Miles, "Capillary-viscous forcing of surface waves," J. Fluid Mech. 219, 635 (1990).

${ }^{13}$ J. W. Miles, "Capillary boundary layer for standing waves," J. Fluid Mech. 222, 197 (1991).

${ }^{14}$ J. W. Miles, "On surface waves with zero contact angle," J. Fluid Mech. 245, 485 (1992).

${ }^{15} \mathrm{C}$. Ting and M. Perlin, "Boundary conditions in the vicinity of the contact line at a vertically oscillating upright plate: An experimental investigation,” J. Fluid Mech. 295, 263 (1995).

${ }^{16}$ G. H. Keulegan, "Energy dissipation in standing waves in rectangular basins," J. Fluid Mech. 6, 33 (1959).

${ }^{17}$ B. Cocciaro, S. Faetti, and C. Festa, "Experimental investigation of capillary effects on surface gravity waves: Non-wetting boundary conditions," J. Fluid Mech. 246, 43 (1993).

${ }^{18}$ L. M. Hocking, "Rival contact-angle models and the spreading of drops," J. Fluid Mech. 239, 671 (1992).

${ }^{19}$ P. Ehrhard and S. H. Davis, "Non-isothermal spreading of liquid drops on horizontal plates," J. Fluid Mech. 65, 81 (1991).

${ }^{20}$ L. Jiang, C. Ting, M. Perlin, and W. W. Schultz, "Moderate and steep Faraday waves: Instabilities, modulation and temporal asymmetries," J. Fluid Mech. 329, 275 (1996).

${ }^{21}$ L. Jiang, M. Perlin, and W. W. Schultz, "Period tripling and energy dissipation of breaking standing waves," J. Fluid Mech. 369, 273 (1998).

${ }^{22}$ D. M. Henderson, J. Hammack, P. Kumar, and D. Shah, "The effects of static contact angles on standing waves," Phys. Fluids A 4, 2320 (1992).

${ }^{23}$ C. Martel and E. Knobloch, "Damping of nearly inviscid water waves," Phys. Rev. E 56, 5544 (1997).

${ }^{24}$ D. M. Henderson and J. W. Miles, "Surface-wave damping in a circular cylinder with a fixed contact line,” J. Fluid Mech. 285, 285 (1994).

${ }^{25}$ T. B. Benjamin and J. C. Scott, "Gravity-capillary waves with edge constraints," J. Fluid Mech. 92, 241 (1979).

${ }^{26}$ M. Perlin and W. W. Schultz, "Capillary effects on surface waves," Annu. Rev. Fluid Mech. 32, 241 (2000).

${ }^{27}$ R. L. Hoffman, "A study of the advancing interface. I. Interface shape in liquid-gas systems," J. Colloid Interface Sci. 50, 228 (1975).

${ }^{28}$ P. G. de Gennes, X. Hua, and P. Levinson, "Dynamics of wetting: Local contact angles,” J. Fluid Mech. 212, 55 (1990).

${ }^{29}$ C. C. Mei and L. F. Liu, "The damping of surface gravity waves in a bounded liquid,’ J. Fluid Mech. 59, 239 (1973).

${ }^{30}$ J. W. Miles, "Surface-wave damping in closed basins," Proc. R. Soc. London, Ser. A 297, 459 (1967). 